\title{
The Influence of Sleep Disturbance and Cognitive Emotion Regulation Strategies on Depressive Symptoms in Breast Cancer Patients
}

\author{
Joohee Lee, MD, Soyoung Youn, MD, Changnam Kim, MD, Sungook Yeo, MD, Seockhoon Chung, MD, PhD \\ Department of Psychiatry, Asan Medical Center, University of Ulsan College of Medicine, Seoul, Korea
}

Received: May 9, 2019

Revised: June 3, 2019

Accepted: June 3, 2019

Correspondence

Seockhoon Chung, MD, PhD

Department of Psychiatry,

Asan Medical Center, University of Ulsan

College of Medicine, 88 Olympic-ro 43-gil,

Songpa-gu, Seoul 05505, Korea

Tel +82-2-3010-3411

Fax +82-2-485-8381

E-mail schung@amc.seoul.kr

ORCID

Joohee Lee

https://orcid.org/0000-0002-3114-1944 Soyoung Youn

https://orcid.org/0000-0003-4983-4017

Changnam Kim

https://orcid.org/0000-0001-6767-696X

Sungook Yeo

https://orcid.org/0000-0003-4127-3040

Seockhoon Chung

https://orcid.org/0000-0002-9798-3642

Background and Objective Depression and insomnia are highly prevalent and important distressing symptoms in breast cancer patients. And also, strategies to assist with cognitive emotions are important to help them cope with the stress of the disease. This study aimed to investigate the effects of sleep disturbance and these coping strategies on depressive symptoms in breast cancer patients.

Methods We retrospectively reviewed the medical records of 119 breast cancer patients. Psychiatric assessments were done using Patient Health Questionnaire-9 (PHQ-9), Insomnia Severity Index (ISI), state subcategory of State and Trait Anxiety Inventory (STAI-S), Cancer-related Dysfunctional Beliefs about Sleep (C-DBS), Fear of Progression (FoP), and Cognitive Emotion Regulation Questionnaire (CERQ).

Results Significant differences in the ISI, C-DBS, FoP, and in the regulation strategies of CERQ, were observed between depressed (PHQ-9 $\geq 10, \mathrm{n}=60$ ) and non-depressed patient groups (PHQ-9 $<10, \mathrm{n}=59, \mathrm{p}<0.05)$. The PHQ-9 score correlated with the ISI, C-DBS, and FoP scores. All maladaptive strategies except blaming others were positively, and most adaptive strategies other than a refocus on planning were negatively correlated with PHQ-9 score $(\mathrm{p}<0.01)$. Linear regression analysis revealed that breast cancer patient depression was predicted by high ISI score, high FoP score, and lower acceptance and higher catastrophizing item scores.

Conclusions Cancer patient depression is associated with insomnia and the cognitive emotion regulation strategies used during their care. Discussions with these patients regarding coping strategies and sleep better will help to improve depressive symptoms. Sleep Med Res 2019;10(1):36-42

Key Words Breast cancer, Adaptation, Depression, Insomnia.

\section{INTRODUCTION}

The WHO has reported that an estimated 627,000 women worldwide died from breast cancer in 2018. In Korea, the national cancer registration statistics (National Cancer Information Center) indicate that breast cancer has the second highest cancer prevalence in women $(197,263)$. A diagnosis of breast cancer is a stressful life event and a substantial percentage of these patients experience depression and anxiety after diagnosis [1]. Previous studies of cancer populations have found that the incidence of psychiatric morbidity in cancer patients differs according to gender and that mood disorders are more common in female cases [2,3]. It is noteworthy also that female patients with breast tumors have the most adjustment issues among various cancer groups. This higher level of distress can be explained by the visibility of these tumors and the very possible loss of a breast [3]. A large-scale five-year observational cohort study reported that nearly $50 \%$ of patients diagnosed with early breast cancer experienced depression and anxiety within one year and 15\% still suffered from depression at the 5-year 
follow-up. The prevalence of pathologic mood symptoms in early breast cancer patients is also reported to be twice as high as that of the general female population [1].

The importance of psychiatric assessment and intervention is emphasized for cancer patients because of the mental health effects of treatment outcomes [4,5]. It is acknowledged that depressed cancer patients have been reported to have poorer adherence to therapy and lower survival rates than non-depressed cancer patients [6]. Early psychiatric evaluation and intervention should therefore be stressed for women diagnosed with breast cancer. Assessment of the influence of sleep disturbance on cancer-related depression is one of the interests of this study. Insomnia is highly prevalent in patients with cancer, affecting up to $50 \%$ of patients [7] and it has been known to be associated with depression severity. Understanding of the relationship between insomnia and depression in cancer patients is important because these two factors appear to increase the risk of cancer morbidity [8]. There is a need for research in insomnia as a potential contributor to patient distress but there is few data in cancer patients to evaluate the association between insomnia and depression.

Interventions and programs to promote positive emotions such as mindfulness meditation and training in relaxation techniques are also important. They have been reported to reduce cancer-related worry, anxiety and depression in patients diagnosed with breast cancer [9]. The management of cognitive emotion is particularly important for cancer patients as they often fall into depression when they fail to cope with stress $[10,11]$. Individual differences exist in the use of specific cognitive emotion regulation strategies [12]. Moreover, the different ways in which patients regulate their emotions in response to external events affects the severity of depressive symptoms. Recent studies have confirmed a strong correlation between cognitive emotional regulation strategies and mood symptoms [13]. Patients using maladaptive strategies such as rumination or catastrophizing were more vulnerable to depression, whereas the application of adaptive strategies such as positive reappraisal served as a defense against this outcome [13-18]. Most studies to date on the relationship between cognitive emotion regulation strategies and depression have focused on the general population and few have reported on patients diagnosed with cancer.

If we can find out how sleep disturbance and coping strategies influence on depressive symptoms of breast cancer patients, it is helpful to manage depressive symptoms of cancer patients for their better quality of life. The aim of our present study was to explore the influence of sleep disturbance and cognitive emotion regulation strategies on depressive symptoms in breast cancer patients.

\section{METHODS}

\section{Participants}

The current study design was a retrospective review of the medical records of breast cancer patients who first visited the psychiatric clinic at Asan Cancer Center. Cancer patients are routinely asked at their first visit to complete an initial questionnaire that assesses their psychological state, including the severity of depressive symptoms, anxiety, insomnia and coping strategies. Among the cancer patients who visited the psychiatric clinic at our hospital between January and July 2017, 119 women with breast cancer aged between 18-80 years had undergone interviews and completed questionnaires on a self-reporting basis and were included as subjects in this study. Breast cancer patients with a communication difficulty, who could not fill out the questionnaires on their own, or who were male were excluded. The medical records for the included cases were reviewed and demographic data were collected including age, cancer stage and treatment types (chemotherapy, radiotherapy, hormonal therapy or surgery). This study was approved by the Institutional Review Board of Asan Medical Center (IRB No. 2018-0529), which waived the requirement for informed consent.

\section{Assessments and Instruments}

The study subject assessment questionnaires included the $\mathrm{Pa}$ tient Health Questionnaire-9 (PHQ-9), Cancer-related Dysfunctional Beliefs about Sleep (C-DBS), Insomnia Severity Index (ISI), state subcategory of State-Trait Anxiety Inventory (STAIS), Fear of Progression (FoP), and Cognitive Emotion Regulation Questionnaire (CERQ).

The PHQ-9 is a reliable and valid self-administered diagnostic tool and was used to assess the severity of depressive symptoms. This tool was designed to match the diagnostic criteria of major depressive disorder and comprises 9 items. It measures the patient's depressive symptoms in the 2 weeks prior to the test. Each item is scored from 0 to 3 for a maximum score of 27 across the 9 items. The cut-off point for depressive symptoms in the PHQ-9 is a score of 10 [19]. A Korean version of the PHQ-9 has been shown to have good reliability and validity [20] and was used for the patients included in this present study.

The CERQ is used to assess the use of cognitive coping strategies when a person experiences an unpleasant event and measures nine different cognitive coping strategies consisting of five adaptive emotion regulation strategies (acceptance, positive refocusing, positive reappraisal, putting into perspective, and refocus on planning) and four maladaptive emotion regulation strategies (self-blame, blaming others, rumination, and catastrophizing). Each strategy refers to the cognitive components showing how a person thinks during a stressful event. The CERQ has shown prospective relationships with depression and anxiety in adults, and its high reliability and validity was confirmed [21]. A Korean version of the CERQ that was 
used for the current study patients has also previously demonstrated good validity [22].

The ISI is a widely used self-reporting questionnaire that comprises 7 items for assessing the severity of insomnia [23]. The cut off for insomnia with this system is a score of 8. A Korean version of the ISI was used for our present study series, as it has demonstrated good validity [24].

The C-DBS is a 2-item tool to measure cancer-related dysfunctional beliefs about sleep. The cancer-related faulty cognitions that play an important role in sleep disturbance are measured with this tool using the responses to 2 questions: "My immune system will have serious problems if I don't go to sleep at a certain time (question 1)" and "If I don't sleep well at night, my cancer can recur or metastasize (question 2)." Each item is scored from 0 to 10 . Higher C-DBS scores are associated with more severe insomnia [25].

The STAI is a tool for measuring the anxiety state. It contains 40 questions that address the patient's feelings about specific situations that are perceived as dangerous (state anxiety) and daily stresses (trait anxiety) [26]. We just used 20 items of state anxiety rated from 1 to 4 , and the total score ranges from 4 to 80 . The Korean version of the STAI was used for our current cases [27].

The FoP is a 43-item tool for evaluating various concerns related to disease progression and has been shown to have good internal consistency and reliability. We used a Korean version of this tool [28] which is a 12-item short form of the original FoP-Q (FoP-Q-SF) that was validated previously in a large sample of breast cancer patients [29]. The 12 FoP-Q-SF items used to evaluate distress symptoms in patients with cancer or other chronic disorders are scored from 1 to 5 per item.

\section{Statistical Analysis}

The t-test was used to compare the demographics and psychological characteristics of the depressed and non-depressed breast cancer patients included in this present study. Chi-square analysis was used to determine the relationship between depression and cancer-related clinical variables, cancer stages and types of treatment (chemotherapy, radiotherapy, and surgery). A Pearson's correlation was used to identify the association between depression and various factors. Based on these results, linear regression was applied to explore the predictive factors for depression in the breast cancer study patients. SPSS version 21.0 software (IBM Corp., Armonk, NY, USA) was used for the statistical analysis. Statistical significance was defined by a p value $<0.05$.

\section{RESULTS}

\section{Demographics and Psychological Characteristics of The Depressed and Non-Depressed Breast Cancer Patients in This Study}

The medical records of 119 women with breast cancer with a mean age of $48.4 \pm 10.5$ years were retrospectively reviewed. Their clinical cancer stages were $0(n=7,5.9 \%), I(n=29,24.4 \%)$, II ( $\mathrm{n}=46,38.7 \%)$, III $(\mathrm{n}=20,16.8 \%)$, and IV ( $\mathrm{n}=17,14.3 \%)$. All of 106 (89.1\%) patients underwent surgical procedure. Subjects were treated with chemotherapy $(n=67,56.3 \%)$, radiation therapy ( $n=44,37.0 \%)$, and hormone therapy $(n=49,41.2 \%)$. These patients were divided into two groups based on the cut-off points of the PHQ-9 scale i.e. depressed (PHQ-9 $\geq 10, \mathrm{n}=60$ ) and non-depressed (PHQ- $9<10, n=59$ ). There was no significant difference in cancer stage, treatment modalities, or psychiatric diagnoses between the depressed and non-depressed groups. Nearly $50 \%$ of the breast cancer patients in our current series had depression. There were no significant differences in age between the depressed and non-depressed groups. Significant differences were observed between these groups in the psychiatric diagnosis, and rating scales scores including ISI, C-DBS and FoP questionnaire scores, and also in the regulation strategies of the CERQ, including putting into perspective, positive refocusing, positive reappraisal, self-blame, rumination and catastrophizing (Table 1).

\section{Correlation between Depressive Symptoms and Other Scales}

Depressive symptoms were found to be significantly correlated with the ISI, CDBS, and FoP but not with the STAI-S (Pearson's correlation, $\mathrm{p}<0.05$ ) (Table 2). The PHQ-9 results in our present patient series were significantly correlated with putting into perspective, positive refocusing, acceptance, positive reappraisal among the adaptive coping skills, and with self-blame, rumination and catastrophizing among the maladaptive coping skills (all, $\mathrm{p}<0.01$ ) (Table 2 ). This same analysis was repeated to detect the relationships between cognitive emotion regulation strategies and other scales. The depressive symptoms of our cancer patients showed a strong association with most of the cognitive strategies. Other emotional symptoms such as fear, dysfunctional belief about cancer, and insomnia were found to be mainly related to the greater use of maladaptive strategies rather than lower use of adaptive strategies. The FoP values significantly correlated with all maladaptive strategies i.e. self-blame, rumination, catastrophizing (all, p < 0.01) and other-blame ( $\mathrm{p}<$ 0.05 ), and with the positive refocusing item of the adaptive strategies $(\mathrm{p}<0.05)$. The C-DBS scores showed a significant correlation with self-blame and catastrophizing $(\mathrm{p}<0.01)$. The ISI scores significantly correlated with catastrophizing $(\mathrm{p}<0.01)$. The state subcategory of the STAI was not significantly correlated with any item of the CERQ.

\section{Predictors of Depression in Women with Breast Cancer}

Our linear regression analysis to explore predictive factors for depression in our breast cancer patients revealed that a high score for the ISI, FoP and catastrophizing item and low score 
Table 1. Demographics and psychological characteristics of the depressed and non-depressed breast cancer patients in this study

\begin{tabular}{|c|c|c|c|}
\hline Variables & Depressed $(n=60)$ & Non-depressed $(n=59)$ & p-value \\
\hline Age & $48.4 \pm 10.4$ & $48.4 \pm 10.5$ & 0.99 \\
\hline \multicolumn{4}{|l|}{ Clinical status } \\
\hline Clinical stages (0/I/II/III/IV), n & $5 / 12 / 24 / 11 / 8$ & $2 / 17 / 22 / 9 / 9$ & 0.65 \\
\hline History of surgical operation & $51(85.0)$ & $55(93.2)$ & 0.24 \\
\hline History of chemotherapy & $34(56.7)$ & $33(55.9)$ & 0.99 \\
\hline History of radiation therapy & $20(33.3)$ & $24(40.7)$ & 0.45 \\
\hline History of hormone therapy & $22(36.7)$ & $27(45.8)$ & 0.36 \\
\hline Psychiatric diagnosis & & & $<0.01$ \\
\hline Normal & $5(8.3)$ & $11(18.65)$ & \\
\hline Adjustment disorder or acute stress reaction & $7(11.7)$ & $7(11.9)$ & \\
\hline Anxiety disorder & $1(1.7)$ & $5(8.5)$ & \\
\hline Depressive disorder & $33(55.0)$ & $8(13.6)$ & \\
\hline Insomnia disorder & $7(11.7)$ & $23(39.0)$ & \\
\hline Mixed anxiety and depressive disorder & $7(11.7)$ & $3(5.1)$ & \\
\hline Others & $0(0.0)$ & $2(3.4)$ & \\
\hline \multicolumn{4}{|l|}{ Rating scales } \\
\hline Patient Health Questionnaire-9 & $15.5 \pm 4.2$ & $6.0 \pm 2.3$ & $<0.01$ \\
\hline Insomnia Severity Index & $17.1 \pm 6.2$ & $13.8 \pm 4.9$ & $<0.01$ \\
\hline Fear of Progression & $44.4 \pm 8.9$ & $31.6 \pm 8.3$ & $<0.01$ \\
\hline State subcategory of State-Trait Anxiety Inventory & $40.4 \pm 6.8$ & $39.4 \pm 7.1$ & 0.45 \\
\hline Cancer-related Dysfunctional Beliefs about Sleep & $13.8 \pm 5.5$ & $11.4 \pm 5.1$ & 0.02 \\
\hline Adaptive cognitive emotion regulation & $25.9 \pm 8.1$ & $30.4 \pm 8.0$ & $<0.01$ \\
\hline Putting into perspective & $5.1 \pm 1.8$ & $5.9 \pm 2.0$ & 0.02 \\
\hline Refocus on planning & $5.9 \pm 2.3$ & $6.4 \pm 1.7$ & 0.27 \\
\hline Positive refocusing & $3.8 \pm 1.5$ & $5.1 \pm 1.6$ & $<0.01$ \\
\hline Acceptance & $6.1 \pm 2.3$ & $6.7 \pm 2.1$ & 0.14 \\
\hline Positive reappraisal & $5.0 \pm 1.9$ & $6.3 \pm 2.3$ & $<0.01$ \\
\hline Maladaptive cognitive emotion regulation & $24.4 \pm 6.0$ & $19.0 \pm 4.8$ & $<0.01$ \\
\hline Self-blame & $6.6 \pm 2.3$ & $5.6 \pm 1.9$ & 0.01 \\
\hline Other-blame & $4.5 \pm 2.4$ & $4.0 \pm 1.7$ & 0.27 \\
\hline Rumination & $6.8 \pm 2.1$ & $5.0 \pm 1.9$ & $<0.01$ \\
\hline Catastrophizing & $6.5 \pm 2.2$ & $4.3 \pm 1.8$ & $<0.01$ \\
\hline
\end{tabular}

for the acceptance item are risk factors for depression $\left(\mathrm{R}^{2}=\right.$ $0.55, \mathrm{p}<0.01$ ) (Table 3). Patients with a high severity of insomnia, more distress symptoms due to fear of disease progression, and a tendency to catastrophize and reject experiences can be expected to have depressive symptoms.

\section{DISCUSSION}

The purpose of our present investigation was to explore the relationships of cognitive emotion regulation strategies, insomnia and depressive symptoms in breast cancer patients. Our findings indicate that insomnia, low acceptance ability, cata- strophizing and fear of disease progression are important predictive factors for the onset of depression in breast cancer patients. The patients who are better able to sleep and cope with negative life events by accepting what has happened and disengaging from catastrophizing are expected to experience fewer depressive symptoms and show better psychological well-being.

We evaluated five scales that would predict depressive symptoms in our breast cancer patients. The ISI and FoP tools have been found to be important predictors of depression. It is well known that depression and insomnia share a bidirectional association. A previous report found that a high ISI score corresponded to a high depression score in cancer sufferers [30]. Previous findings with these systems line are consistent with 
Table 2. Correlation between depressive symptoms and other scales

\begin{tabular}{|c|c|c|c|c|c|}
\hline \multirow{2}{*}{ Variables } & \multicolumn{5}{|c|}{ Pearson's coefficient $(\mathrm{r})$} \\
\hline & PHQ-9 & C-DBS & ISI & FoP & STAI-S \\
\hline Age & 0.06 & -0.03 & -0.03 & -0.14 & 0.14 \\
\hline PHQ-9 & - & $0.20^{\dagger}$ & $0.32^{+}$ & $0.63^{\dagger}$ & 0.15 \\
\hline \multicolumn{6}{|c|}{ Adaptive cognitive emotion regulation } \\
\hline Putting into perspective & $-0.25^{\dagger}$ & -0.03 & -0.03 & -0.09 & 0.03 \\
\hline Refocus on planning & -0.14 & 0.10 & 0.12 & 0.11 & 0.03 \\
\hline Positive refocusing & $-0.37^{\dagger}$ & -0.10 & -0.12 & $-0.20^{*}$ & 0.01 \\
\hline Acceptance & $-0.26^{\dagger}$ & -0.01 & 0.05 & 0.02 & -0.02 \\
\hline Positive reappraisal & $-0.30^{\dagger}$ & 0.07 & -0.06 & -0.11 & -0.01 \\
\hline \multicolumn{6}{|c|}{ Maladaptive cognitive emotion regulation } \\
\hline Self-blame & $0.26^{\dagger}$ & $0.24^{\dagger}$ & 0.06 & $0.31^{\dagger}$ & -0.07 \\
\hline Other-blame & 0.17 & 0.14 & 0.16 & $0.19^{*}$ & 0.18 \\
\hline Rumination & $0.51^{\dagger}$ & 0.06 & 0.18 & $0.44^{\dagger}$ & 0.16 \\
\hline Catastrophizing & $0.57^{\dagger}$ & $0.28^{\dagger}$ & $0.25^{\dagger}$ & $0.55^{\dagger}$ & 0.16 \\
\hline
\end{tabular}

${ }^{*} \mathrm{p}<0.05 .{ }^{\dagger} \mathrm{p}<0.01$.

PHQ-9: Patient Health Questionnaire-9, C-DBS: Cancer-Related Dysfunctional Beliefs about Sleep, ISI: Insomnia Severity Index, FoP: Fear of Progression, STAI-S: state subcategory of the State-Trait Anxiety Inventory.

Table 3. Result of linear regression analysis for expecting depression

\begin{tabular}{clrrrr}
\hline Outcome: depression & Explanatory variables & $\beta$ & $\mathrm{B}$ & $\mathrm{SE}$ & $\mathrm{p}$-value \\
\hline PHQ-9 $\left(\mathrm{R}^{2}=0.55, \mathrm{p}<0.01\right)$ & Acceptance & -0.25 & -0.65 & 0.16 & $<0.01$ \\
& Catastrophizing & 0.24 & 0.62 & 0.20 & $<0.01$ \\
& Insomnia Severity Index & 0.18 & 0.18 & 0.07 & $<0.01$ \\
& Fear of Progression & 0.47 & 0.25 & 0.04 & $<0.01$ \\
\hline
\end{tabular}

B: standardized coefficients, B: un-standardized regression coefficients, SE: standard error.

our current results, i.e. a high FoP score was found to positively correlate with anxiety and depression in cancer patients [28]. Early screening of depression could be done with a simple set of questionnaires. These tools are self-reporting and thus can be completed by patients on their own. The patients with high ISI and FoP scores and that show a dysfunctional use of cognitive emotional regulation strategies could thereby be readily identified. This simple evaluation will also be very helpful for the active cooperation between oncology and psychiatry specialists, and therefore improve the approaches used for the initiation of psychiatric interventions in cancer cases. It is also helpful to engage with patients on their use of coping strategies and help them to sleep better and control their fear of progression.

Consistent with our present study observations, many prior general population studies have reported that cognitive emotion regulation strategies are significantly related to depression $[12,15,18]$. In particular, catastrophizing, rumination, and positive reappraisal have been the most significant factors described in most studies to date $[10,13,14,16,17]$. Similarly, rumination and catastrophizing have shown a strong association with depression in cancer patients and positive refocusing has been found to help cancer patients to better recover from emotional stress [4]. Others of the nine cognitive emotion regulation strategies have also shown a significant association with depression, but to a varying degree between studies.

Little is currently known about the effects of cognitive emotion regulation strategies on depressive symptoms in Asian women with breast cancer. In our current analyses, the value of acceptance and catastrophizing strategies were considered to be powerful predictors of depression, consistent with previous research in a Chinese population [31]. In that prior study, Li et al. [31] reported that catastrophizing as well as acceptance were the best predictors of the patient's ability to adjust to stress. Acceptance refers to accepting what one has experienced and resigning oneself to what has happened. Accepting a situation for what it is allows the active management of emotions rather than being overwhelmed by distressing emotions. Therefore, a patient who accepts the reality of a stressful situation will likely engage in the attempts to deal with the problem. Acceptance has been reported to have positive associations with a positive reinterpretation of a stressful situation and to help patients with psychological adjustment and a better perceived quality of life. Catastrophizing refers to thoughts that explicitly emphasize the terror of a stressful experience and has been consistently report- 
ed to have a positive relationship with more severe depression, anxiety and poorer perception of quality of life [14,31]. Greater use of catastrophizing and less use of acceptance will lead to a poor adjustment to stress events.

Interventions that modify the dysfunctional use of cognitive emotion regulation may play an important role in preserving the psychological well-being of cancer patients. To our knowledge, there is a current lack of research on the outcomes of psychiatric interventions that target the distinct acceptance and catastrophizing strategies. However, as mentioned earlier, several studies on altering the general use of coping strategies in breast cancer patients have reported that the intervention participants had greater coping efficacy, lower depressive symptoms and less perceived stress [9]. Hamama-Raz et al. [32] reported that appropriate interventions during cancer treatment changed the pattern of use of coping strategies and that the patients then experienced more positive long term outcomes in terms of the enhancement of positive strategies and reduction of negative coping strategies.

There were several limitations to this study of note. First, we did not consider all of the risk and protective factors for depression. Several demographic factors such as a prior divorce, low socioeconomic status, lack of intimate relationships and a family history of psychiatric disease are known to be important risk factors for depression. We had limited data on these demographic factors however in our study population. Second, our current data were based on self-reported assessment scales. The evaluations of symptoms in our study cases were scored from subjective assessments and did not include the observations of others. Self-reporting can contain a degree of exaggeration or underestimation. Third, male breast cancer patients were excluded from this study. While breast cancer is predominantly gender-specific, a few male cases do exist. The exploration of the gender effects on the use of cognitive emotion regulation strategies is therefore limited in this type of cancer.

Despite these limitations, our current study findings highlight the potential importance of psychological intervention for women with breast cancer. The prevalence of depression in these cases shows a relationship with the use of cognitive emotion regulation strategies, the severity of sleep disturbance, and fear of disease progression, rather than with cancer-related clinical factors such as the types of treatment or stage of cancer. Improvements in coping skills and appropriate management of insomnia and fear of progression will likely lead to a reduction in depressive symptoms and positive therapeutic outcomes in this population. The development of appropriate cognitive interventions to assist breast cancer patients to acquire the coping skills to manage disease-related stress is warranted. Further studies of the cognitive interventions required to modify maladaptive coping skills in this patient population are also needed.

\section{Conflicts of Interest}

The authors have no financial conflicts of interest.

\section{Authors' Contribution}

Conceptualization: Chung S, Youn S. Data curation: Chung S, Youn S, Kim C, Yeo S. Formal analysis: Lee J, Chung S. Methodology: Lee J, Chung S. Project administration: Lee J. Resources: Chung S, Youn S. Software: Lee J, Kim C, Yeo S. Supervision: Chung S. Validation: Chung S. Visualization: Lee J, Yeo S. Writing-original draft: Lee J. Writing-review \& editing: Chung S, Youn S.

\section{REFERENCES}

1. Burgess C, Cornelius V, Love S, Graham J, Richards M, Ramirez A. Depression and anxiety in women with early breast cancer: five year observational cohort study. BMJ 2005;330:702.

2. Harrison J, Maguire P. Predictors of psychiatric morbidity in cancer patients. Br J Psychiatry 1994;165:593-8.

3. Liang LP, Dunn SM, Gorman A, Stuart-Harris R. Identifying priorities of psychosocial need in cancer patients. Br J Cancer 1990;62:1000-3.

4. Schroevers M, Kraaij V, Garnefski N. How do cancer patients manage unattainable personal goals and regulate their emotions? $\mathrm{Br} J$ Health Psychol 2008;13:551-62.

5. Irwin MR. Depression and insomnia in cancer: prevalence, risk factors, and effects on cancer outcomes. Curr Psychiatry Rep 2013;15:404.

6. DiMatteo MR, Haskard-Zolnierek KB. Impact of depression on treatment adherence and survival from cancer. In: Kissane DW, Ma M, Sartorius N (Eds.), World Psychiatric Association, Depression and cancer 2011;101-24.

7. O’Donnell JF. Insomnia in cancer patients. Clin Cornerstone 2004;6 Suppl 1D:S6-14

8. Sigurdardottir LG, Valdimarsdottir UA, Fall K, Rider JR, Lockley SW, Schernhammer E, et al. Circadian disruption, sleep loss, and prostate cancer risk: a systematic review of epidemiologic studies. Cancer Epidemiol Biomarkers Prev 2012;21:1002-11.

9. Cameron LD, Booth RJ, Schlatter M, Ziginskas D, Harman JE. Changes in emotion regulation and psychological adjustment following use of a group psychosocial support program for women recently diagnosed with breast cancer. Psychooncology 2007;16:171-80.

10. Stikkelbroek Y, Bodden DHM, Kleinjan M, Reijnders M, van Baar AL. Adolescent depression and negative life events, the mediating role of cognitive emotion regulation. PLOS ONE 2016;11:e0161062.

11. Garnefski N, Kraaij V, Schroevers MJ, Aarnink J, van der Heijden DJ, van Es SM, et al. Cognitive coping and goal adjustment after first-time myocardial infarction: relationships with symptoms of depression. Behav Med 2009;35:79-86.

12. John OP, Gross JJ. Healthy and unhealthy emotion regulation: personality processes, individual differences, and life span development. J Pers 2004;72:1301-33.

13. Garnefski N, Teerds J, Kraaij V, Legerstee J, van den Kommer T. Cognitive emotion regulation strategies and depressive symptoms: differences between males and females. Pers Individ Diff 2004;36:267-76.

14. Garnefski N, Kraaij V, Spinhoven P. Negative life events, cognitive emotion regulation and emotional problems. Pers Individ Diff 2001;30: 1311-27.

15. Anderson CA, Miller RS, Riger AL, Dill JC, Sedikides C. Behavioral and characterological attributional styles as predictors of depression and loneliness: review, refinement, and test. J Pers Soc Psychol 1994;66: 549-58.

16. Garnefski N, Kraaij V. Relationships between cognitive emotion regulation strategies and depressive symptoms: a comparative study of five specific samples. Pers Individ Diff 2006;40:1659-69.

17. Schroevers M, Kraaij V, Garnefski N. Goal disturbance, cognitive coping strategies, and psychological adjustment to different types of stressful life event. Pers Individ Diff 2007;43:413-23. 
18. Gross JJ, John OP. Individual differences in two emotion regulation processes: implications for affect, relationships, and well-being. J Pers Soc Psychol 2003;85:348-62.

19. Kroenke K, Spitzer RL, Williams JB. The PHQ-9: validity of a brief depression severity measure. J Gen Intern Med 2001;16:606-13.

20. Choi HS, Choi JH, Park KH, Joo KJ, Ga H, Ko HJ, et al. Standardization of the Korean Version of Patient Health Questionnaire-9 as a Screening Instrument for Major Depressive Disorder. J Korean Acad Fam Med 2007;28:114-9.

21. Garnefski N, Kraaij V. The Cognitive Emotion Regulation Questionnaire: psychometric features and prospective relationships with depression and anxiety in adults. Eur J Psychol Assess 2007;23:141-9.

22. Ahn HN, Lee NB, Joo HS. Validation of the Cognitive Emotion Regulation Questionnaire in a Korean population. Korean J Counsel 2013; 14:1773-94.

23. Bastien $\mathrm{CH}$, Vallières $\mathrm{A}$, Morin CM. Validation of the Insomnia Severity Index as an outcome measure for insomnia research. Sleep Med 2001; 2:297-307.

24. Cho YW, Song ML, Morin CM. Validation of a Korean version of the insomnia severity index. J Clin Neurol 2014;10:210-5.

25. Chung S, Youn S, Choi B. Assessment of cancer-related dysfunctional beliefs about sleep for evaluating sleep disturbance in cancer patients. Sleep Med Res 2017;8:98-101.

26. Metzger RL. A reliability and validity study of the State-Trait Anxiety Inventory. J Clin Psychol 1976;32:276-8.

27. Hahn DW, Lee CH, Chon KK. Korean adaptation of Spielberger's STAI (K-STAI). Korean J Health Psychol 1996;1:1-14.

28. Shim EJ, Shin YW, Oh DY, Hahm BJ. Increased fear of progression in cancer patients with recurrence. Gen Hosp Psychiatry 2010;32:169-75.

29. Mehnert A, Berg P, Henrich G, Herschbach P. Fear of cancer progression and cancer-related intrusive cognitions in breast cancer survivors. Psychooncology 2009;18:1273-80.

30. Seyidova Khoshknabi D, Bennani-Baiti N, Kirkova J, Lasheen W, Davis $\mathrm{M}$, Walsh D, et al. Insomnia in advanced cancer. J Palliat Care 2008; 24:204.

31. Li L, Zhu X, Yang Y, He J, Yi J, Wang Y, et al. Cognitive emotion regulation: characteristics and effect on quality of life in women with breast cancer. Health Qual Life Outcomes 2015;13:51.

32. Hamama-Raz Y, Pat-Horenczyk R, Perry S, Ziv Y, Bar-Levav R, Stemmer SM. The effectiveness of group intervention on enhancing gognitive emotion regulation strategies in breast cancer patients: a 2-year follow-up. Integr Cancer Ther 2016;15:175-82. 